Compres. Countermeasures involving sensory stimulation for the treatment of Jacksonian seizures have been advocated for centuries, dating back to the time of Galen and Aretaeus. Brown-Séquard (1872) proposed an encircling blister and Gowers (1901) found that forcing the closed fingers to open or preventing a leg from flexing would break up a motor march (quoted in Lennox WG. Epilepsy and Related DIsorders Vol 1, 1960, Little, Brown \& Co, Boston). The value of these methods in the treatment of other seizure types should be explored further.

\title{
BEHAVIORAL COMPLICATIONS OF FRONIAL LOBE SEIZURES
}

A 13-yr-old girl with marked behavioral and selective cognitive deficits associated with partial complex seizures of frontal lobe origin is reported from the Depts of Neurology, Psychiatry and Pediatrics, Harbor-UCLA Medical Center, $1000 \mathrm{~W}$ Carson St, Torrance, CA. Behavioral deterioration consisting of inattention, sexual disinhibition, loss of concern for personal hygiene, physical and verbal aggression and periodic incoherent and bizarre speech, was concurrent with a 6-week history of seizures manifested by turning to the right, staring, picking at her clothes, shaking and urinary incontinence. An EEG showed periodic bursts of $2 \frac{1}{2} \mathrm{~Hz}$ spike and slow wave complexes originating in the left frontal lobe. CT and MRI of the brain were normal. Neuropsychological testing for frontal lobe dysfunction (finger tapping, digit span, trailmaking, WISC-R mazes, Stroop test) demonstrated deficits in attention, response inhibition, alteration between tasks, maze solving, word generation and motor functioning. Overall intelligence, basic language skills, visual perception, constructional ability and remote memory were spared. Seizures were controlled, the EEG became normal, and the cognitive and behavioral deficits disappeared within one month after treatment with carbamazepine. On re-examination, the patient was composed, quiet and well groomed, and the content of her speech was appropriate, including memory and expression of shame regarding her behavior during preceding months. (Boone KB et al. Neuropsychological and behavioral abnormalities in an adolescent with frontal lobe seizures. Neurology April 1988; 38: 583-586).

COMirNT. This case-report suggests that frontal lobe seizures and interictal dysfunction may be associated with acute psychiatric disturbance in children. Whereas this patient exhibited remarkable reversible behavioral and cognitive defects without an underlying structural cerebral lesion, in some adults with destructive and selective frontal lobe pathology neuropsychological testing has failed to demonstrate behavioral deficits. It is possible that the seizure activity in the present case may have spread to involve areas in addition to the frontal lobe.

\section{SEIZURES DURING FXXRCISE}

Three children with epileptic seizures occurring during exercise are reported from the section of Electroencephalography and Pediatric Neurology, Mayo Clinic, Rochester, MN. Epileptiform paroxysms were noted with exercise but not with hyperventilation during EEG recordings in these patients. The pathophysiologic mechanism was unclear. (ogunyemi AO et 
al. Seizures induced by exercise. Neurology April 1988; 38: 633-634).

COMENT. A cardiac pathogenesis is usually suspected when seizures occur during physical exercise, as noted by the authors. A full cardiac examination including EKG and echocardiogram is advisable in patients with seizures occurring during physical exertion, even in children. Exercise is an unusual precipitant of seizures and is generally accompanied by a reduction in EEG seizure discharges. A clinical seizure was not induced but a spike and wave

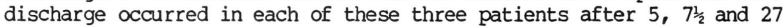
minutes of pedaling a stationary bicycle.

\section{CORN OII KETOGENIC DIET}

The successful substitution of corn oil for MCT oil in 6 children treated with the ketogenic diet for intractable seizures is reported from the Depts of Pediatrics, Neurology and Psychiatry, University of Arkansas for Medical Sciences and Arkansas Children's Hospital, 804 Wolfe St., Little Rock, AR. Seizure types were mixed in all 6 patients, absence in 5, minor motor in 4, myoclonic in 3, and complex partial and generalized tonic-clonic in 1. All had been controlled with MCT oil diets but corn oil has the major advantages of being less expensive, more readily available without prescription, and better tolerated. Anticonvulsants were reduced in 5 patients and eliminated in 3, without deterioration in seizure control. (Woody RC et al. Corn oil ketogenic diet for children with intractable seizures. J Child Neurol Jan 1988; $\underline{3}$ : 21-24.

COMENT. The medium chain triglyceride (MCT) oil was advocated by Huttenlocher et al. (Neurology 1971; 21: 1097) as a substitute for dietary fats in the ketogenic diet. MCT's are more ketogenic and less restrictive of carbohydrates, they are more rapidly absorbed than dietary fat and may induce ketosis more quickly. A disadvantage of the MCT diet is the frequency of gastrointestinal side-effects, many patients suffering from bulky, loose stools, diarrhea, vomiting and abdominal pain. Perhaps the superiority and availability of corn oil will encourage a renewed interest in the ketogenic diet for the treatment of refractory seizures in children:

\section{FOCAL MYOCLONUS AND CORITCAL DYSPLASIA}

Four patients aged 9 to 19 years with focal myoclonus and intractable focal motor seizures beginning at age 4 to 6 years were treated surgically at the Montreal Neurological Hospital and Institute, MoGill University, Montreal, Quebec, Canada. All 4 had a mild progressive hemiparesis, cognitive deficits, focal EEG seizure discharges in the contralateral rolandic areas, 3 showed cortical architectonic abnormalities on MRI, and evidence of focal cortical dysplasia with abnormally wide gyri at operation. All improved following surgery. The authors stress the value of the MRI in visualization and diagnosis of neuronal migration cortical dysplasias whereas other imaging techniques had been unrevealing. (Kuzniecky R, Berkovic S. Andermann F (correspondent) et al. Focal cortical myoclonus and rolandic cortical dysplasia; clarification by magnetic resonance imaging. Ann Neurol April 1988; 23: 317-325).

COMENI. The authors consider the clinical, MRI and pathological 